\title{
Origin of the Growing Length Scale in $M$-p-Spin Glass Models
}

\author{
Joonhyun Yeo ${ }^{1,2, \text { f }}$ and M. A. Moore ${ }^{3, \dagger}$ \\ ${ }^{1}$ Division of Quantum Phases and Devices, School of Physics, Konkuk University, Seoul 143-701, Korea \\ ${ }^{2}$ School of Physics, Korea Institute for Advanced Study, Seoul 130-722, Korea \\ ${ }^{3}$ School of Physics and Astronomy, University of Manchester, Manchester M13 9PL, UK
}

(Dated: September 2, 2021)

\begin{abstract}
Two versions of the $M$ - $p$-spin glass model have been studied with the Migdal-Kadanoff renormalization group approximation. The model with $p=3$ and $M=3$ has at mean-field level the ideal glass transition at the Kauzmann temperature and at lower temperatures still the Gardner transition to a state like that of an Ising spin glass in a field. The model with $p=3$ and $M=2$ has only the Gardner transition. In the dimensions studied, $d=2,3$ and 4 , both models behave almost identically, indicating that the growing correlation length as the temperature is reduced in these models - the analogue of the point-to-set length scale - is not due to the mechanism postulated in the random first order transition theory of glasses, but is more like that expected on the analogy of glasses to the Ising spin glass in a field.
\end{abstract}

PACS numbers: 64.70.Q-, 75.10.Nr, 11.10.Hi, 75.40.Cx

One of the leading contenders for a theory of glasses is the random first-order transition theory (RFOT) [1 3 . It had its genesis in $p$-spin glass models [1. The particular $p$-spin models which might be relevant to the properties of structural glasses have a mean-field limit in which there are two critical temperatures $T_{d}$ and $T_{K}$. The upper temperature $T_{d}$ marks the temperature at which dynamical singularities appear and are like those found in mode-coupling theory [4]. The lower temperature $T_{K}$ is the temperature of the ideal glass transition. This occurs where the configurational entropy (or log of the number of metastable states) vanishes [5. Mean-field like calculations on glass-forming liquid models support this picture [6, 7].

It has always been recognized that the dynamical transition at $T_{d}$ will disappear outside the mean-field limit due to activated processes out of the metastable states. These activated processes make even the existence of metastable states problematical. In a recent paper Franz et al. 8, calculated a dynamical correlation length using a field theoretic approach and found on comparing with numerical data that the agreement was only good when the length scale was of the order of a particle diameter. The field theory predicts that this length scale should diverge but the simulations reveal that the correlation length remains small [9, even though time scales increase rapidly: the dynamical transition is an example of an avoided transition.

The ideal glass transition at $T_{K}$ at mean-field level (or infinite dimension) is associated with a static (equilibrium) transition to a state with one-step replica symmetry breaking (1RSB) [1. The order parameter $q$ jumps from zero in the high temperature phase to a finite value at and below $T_{K}$. It is this jump in $q$ which leads to the "first-order" part in the name of the RFOT theory. The

\footnotetext{
* jhyeo@konkuk.ac.kr

$\dagger$ m.a.moore@manchester.ac.uk
}

configurational entropy (or complexity) vanishes as the temperature approaches $T_{K}$ from above. While there is widespread agreement that the transition at $T_{d}$ becomes just a crossover or avoided transition in finite dimensions, there is no consensus about what happens to the ideal glass transition outside the mean-field limit. One of us has argued that the 1RSB transition must also be avoided in any finite dimension [10, just like the dynamical transition at $T_{d}$. In other words, the lower critical dimension $d$ of the 1RSB state is infinite.

In this paper we examine $M$ - $p$-spin models; in particular the cases of $p=3$ with $M=2$ and $M=3$. These variants of the $p$-spin model have been extensively studied [11-15]. Their significance is that calculations and simulations can be done with them both at the mean-field level and in finite dimensions. In the $M$ - $p$-spin model, there are $M$ Ising spins $\sigma_{i}^{(\alpha)}, \alpha=1,2, \cdots, M$ on each site $i$ of (say) a hypercubic lattice. The spins interact with each other via a $p$-body interaction. The Hamiltonian involves terms of products of $p$ spins chosen from the spins in a pair of nearest-neighbor sites. For the $p=3$ case, the Hamiltonian is given by

$$
\begin{aligned}
\mathcal{H}=-\sum_{\langle i j\rangle} \sum_{\alpha<\beta}^{M} \sum_{\gamma}^{M} & \left(J_{i j}^{(\alpha \beta) \gamma} \sigma_{i}^{(\alpha)} \sigma_{i}^{(\beta)} \sigma_{j}^{(\gamma)}\right. \\
& \left.+J_{i j}^{\gamma(\alpha \beta)} \sigma_{i}^{(\gamma)} \sigma_{j}^{(\alpha)} \sigma_{j}^{(\beta)}\right),
\end{aligned}
$$

where the notation $\langle i j\rangle$ means that the sum is over all nearest neighbor pairs $i$ and $j$. The number of different coupling constants, $J_{i j}^{(\alpha \beta) \gamma}$ and $J_{i j}^{\gamma(\alpha \beta)}$ for given $\langle i j\rangle$ is just $2 M\left(\begin{array}{c}M \\ 2\end{array}\right)=M^{2}(M-1)$. All these couplings are usually chosen independently from a Gaussian distribution with zero mean and width $J$.

The versions with $M=2$ and $M=3$ are of particular interest as at mean-field level they have quite different kinds of behavior. The model with $M=3$ at meanfield level has both a dynamical transition at $T_{d}$ and an ideal glass transition at $T_{K}$. The model with $M=2$ is 
completely different at mean-field level. It has neither of these transitions. The origin of the differences can be glimpsed by putting the $M$ - $p$-spin model into a field theoretical framework.

The standard way of doing this is to use the HubbardStratonovich transformation on the replicated partition function and then trace over the spins. The resulting field theory associated with this model is the following Ginzburg-Landau-Wilson Hamiltonian

$$
\begin{aligned}
\mathcal{H}_{\mathrm{GLW}}=\int d^{d} \mathbf{r} & \left\{\frac{1}{2} \sum_{a<b}\left[\nabla q_{a b}(\mathbf{r})\right]^{2}+\frac{t}{2} \sum_{a<b} q_{a b}^{2}(\mathbf{r})\right. \\
& \left.-\frac{w_{1}}{6} \operatorname{Tr} q^{3}(\mathbf{r})-\frac{w_{2}}{3} \sum_{a<b} q_{a b}^{3}(\mathbf{r})\right\}
\end{aligned}
$$

where $q_{a b}(\mathbf{r})$ is the order parameter and $a$ and $b$ are replica indices running from 1 to $n$ with $n \rightarrow 0$. At mean field level, this model has been known for a long time to show very different behavior depending on the value of $R=w_{2} / w_{1}[16$. When $R>1$, there are two transitions at the mean field level as described above; a dynamical transition at some temperature $T_{d}$ and a thermodynamic transition at a lower temperature $T_{K}$ to a state with onestep replica symmetry breaking. When $R<1$ neither of these transitions will occur. In Ref. [17, the ratio $R$ was evaluated for the $M-p$-spin model for general values of $M$ and $p$. The cases we are interested in this paper, namely $p=3, M=2$ and $p=3, M=3$, correspond to $R \approx 0.879$ and $R=2$, respectively. Therefore the two models should indeed show very different mean-field behavior.

At temperatures below $T_{K}$ for the case where $R>1$, there is yet another transition, the Gardner transition, to a state with full replica symmetry breaking (RSB) [18. For the case where $R<1$, there is at mean-field level only one transition - the Gardner transition, a continuous transition to a state which is expected to have full RSB, (although this has never been checked explicitly to the best of our knowledge).

The transition discovered by Gardner is thus present for both the $M=2$ and $M=3$ models at mean-field level [18]. She showed that the state with full replica symmetry breaking was very similar to that of the Ising spin glass in an applied field $h$. For this model, there is a line in the $h-T$ phase diagram, the de Almeida-Thouless (AT) line, 19], which separates the paramagnetic replica symmetric state from the state with full replica symmetry breaking. Arguments have been presented [20, 21] that the lower critical dimension of states with full replica symmetry breaking is 6 . The Gardner transition, which is in the same universality class as the AT transition, should be another avoided transition for all $d \leq 6$.

In this paper we have studied both the models with $M=2$ and $M=3$ within the Migdal-Kadanoff (MK) renormalization group approximation in dimensions $d=$ 2, 3 and 4 to determine how thermal fluctuations modify the mean-field picture of these two models. The MK
TABLE I. The correlation length $\xi(0)$ in the zerotemperature limit for various values of $M$ and $d$ measured in units of the lattice spacing.

\begin{tabular}{lccc}
\hline \hline & $d=2$ & $d=3$ & $d=4$ \\
$M=2$ & 11 & 26 & 72 \\
$M=3$ & 12 & 38 & 165 \\
\hline \hline
\end{tabular}

approximation is one of the few approximations which is reliable for the study of spin glasses in low dimensions [11, 12. The details of our calculation are as in [11, 12. We are interested in particular whether in the physically relevant dimensions, $d=2$ and $d=3$, whether there are any vestiges left of the mean-field transitions. One can see in the molecular dynamics study of Kob et al. [9], clear remnants of the dynamical transition. Only equilibrium properties are studied in this paper so only the remnants of transitions which could be seen are those of the ideal glass transition and the Gardner transition for the case with $M=3$, and just the Gardner transition for the case $M=2$.

We determined the correlation length $\xi$ by the same method which was used in Refs. [11, 12, 15], that is via the decay of the interactions $J_{i j}$ with distance $L$ on the MK hierarchical lattice: $J_{i j} \sim \exp (-L / \xi)$. As the temperature $T$ is reduced to zero this correlation length grows to a value $\xi(0)$, which is strikingly large especially for $d=4$. The data on $\xi(0)$ are presented in Table I] The large values of $\xi(0)$ certainly suggests there is an avoided transition mechanism at work. Even in $d=3, \xi(0)$ is considerably larger than those which have been obtained in simulations of realistic glass models, at least down to temperatures which are currently practical 9 .

It is useful to measure temperature $T$ on the scale of the mean-field transition temperature $T_{c}$ (defined as when the coefficient $t$ in Eq. (2) equals zero). For $M=2$, $T_{c}=(\sqrt{2} z)^{1 / 2} J$, while for $M=3, T_{c}=(3 z)^{1 / 2} J$, where $z=2 d$ is the number of nearest neighbors on the hypercubic lattice [17]. In Fig. 1 the ratio of $\xi / \xi(0)$ has been plotted as a function of $T / T_{c}$. It shows that as a function of $T / T_{c}$, the ratio $\xi / \xi(0)$ is essentially the same for both $M=2$ and $M=3$. We had expected to see for the case of $M=3$ features which could be associated with a possibly avoided ideal glass transition at $T_{K}$. None is visible in Fig. 1. This result is our main finding. What is its significance?

The correlation length studied here is the equivalent of the point-to-set length scale [9, 22, 23, studied in glassy supercooled liquids. In the RFOT theory [2, 3, this length scale grows as the temperature is reduced and eventually diverges at $T_{K}$. In that theory the growth of the correlation length is driven by the decrease of the configurational entropy (or complexity) to zero as the temperature approaches $T_{K}$. Since for $M=2$ there is no ideal glass transition yet this model is almost identical in its properties with those of the $M=3$ model, the growing 


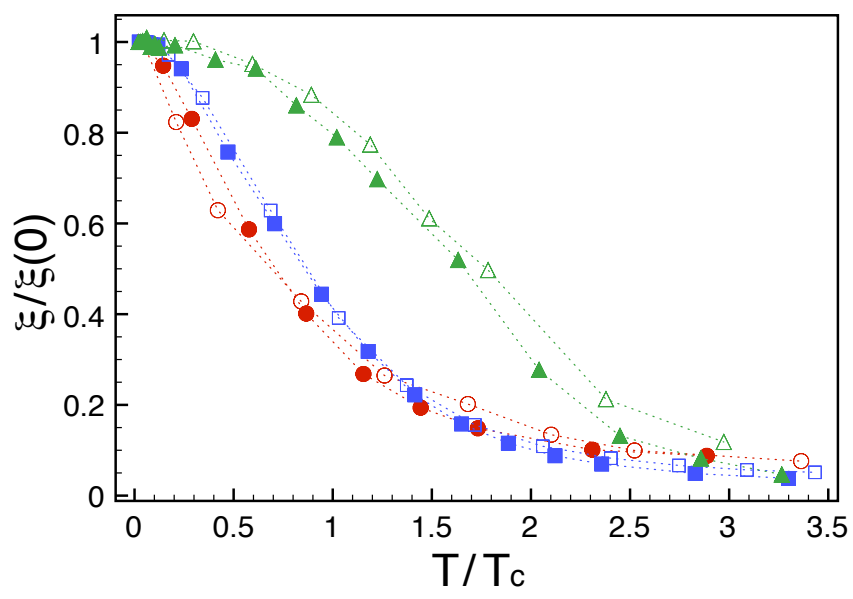

FIG. 1. (Color online) Behavior of the scaled correlation function $\xi / \xi(0)$ plotted versus the scaled temperature $T / T_{c}$. The closed symbols correspond to $M=3$, the open symbols to $M=2$. The upper (green triangles) curves are for $d=4$, the middle curves (blue squares) are for $d=3$, while the lower two curves (red circles) are for $d=2$.

correlation length cannot be a remnant of the ideal glass transition. It must instead be a remnant of the Gardner transition which is common to both models.

Thus the mechanism behind the growing correlation length as the temperature is reduced cannot be that envisaged in the RFOT, but instead must be that associated with the growing correlation length which arises in the Ising spin glass in a field as the temperature is lowered. According to the droplet picture, [24 26] the correlation length increases as the temperature is decreased and saturates at $T=0$ to a value set by equating the interface energy between a droplet of size $\xi(0)$ and its time reverse, $\sim J \xi(0)^{\theta}$, to the energy gained from the field on flipping the droplet, $\sim h \xi(0)^{d / 2}$. The exponent $\theta \approx-0.28$ for $d=2$ and $\theta \approx 0.24$ for $d=3$ (see Ref. [27. for a review of the value of $\theta$ in various dimensions $d)$. Table 1 shows that $\xi(0)$ gets larger as the dimensionality goes up and when there is an AT line, i.e. when $d>6$, it would be expected to be infinite. The MK approximation itself is a low-dimensional approximation (it is exact in one dimension), and cannot be trusted to be even qualitatively correct in dimensions as high as six.

We have argued before that the growing (point-to-set) length scale seen in supercooled liquids as the temperature is reduced [28, 29] is a consequence of their being in the same "universality class" as the Ising spin glass in a field. However, until now, we could not rule out the possibility that the growing length scale might arise through a 1RSB transition as in RFOT, (but possibly avoided because of the mechanism in Ref. [10]). The similarity between the $M=2$ and $M=3$ models shown in Fig. 1 now removes that possibility for the $M=3$ model. In a recent paper 30, it has been argued that the discontinuous 1RSB transition in the $M=3$ model might be

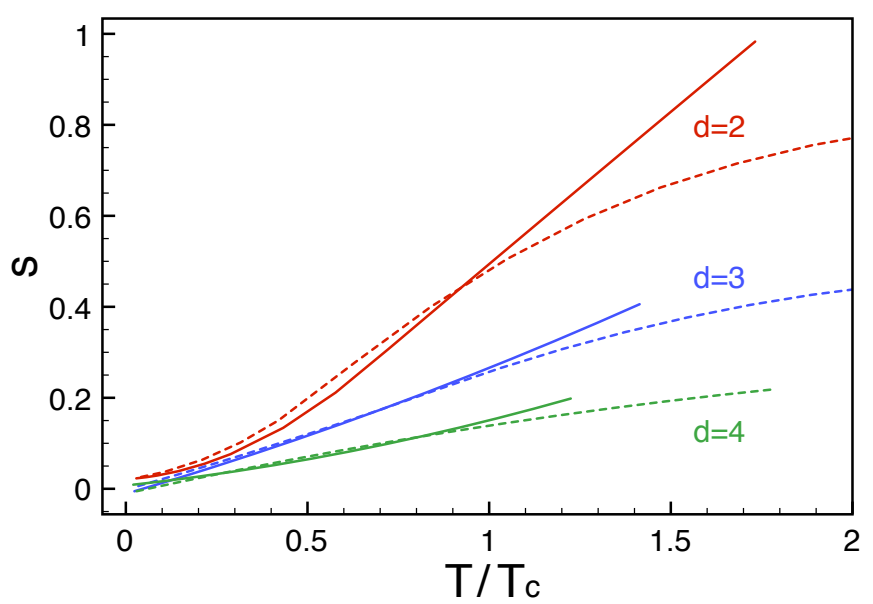

FIG. 2. (Color online) The entropy $S$ per site as a function of the scaled temperature $T / T_{c}$. The solid lines are for the $M=3$ model, the dashed lines are for the $M=2$ model.

removed by the fluctuations on short length scales.

In RFOT theory, the configurational entropy is argued to go to zero at the Kauzmann temperature $T_{K}$. For $M-p$ spin glass models it is not clear how the configurational entropy should be determined outside the meanfield limit, but we have studied their total entropy by numerically differentiating the free energy calculated within the MK approximation (which leads to some inaccuracy near $T=0$ ). It is plotted in Fig. 2 as a function of the reduced temperature $T / T_{c}$. Once again the models with $M=2$ and $M=3$ behave almost identically, indicating that when the correlation length gets large, there is present a form of universality. At high temperatures where the correlation length is small, the two types of model have very different entropies: the high temperature limit of the entropy per site is $k_{B} M \ln 2$. While there is no sign in Fig. 2 of the entropy vanishing below some temperature $T_{K}$, the entropy is smaller at lower temperatures for the $d=4$ versions of the model, and behavior in four dimensions is going to be closer to mean-field theory than behavior in two dimensions.

We have also determined the Edwards-Anderson order parameter $q$

$$
q=\left[\left\langle\sigma_{i}^{(\alpha)}\right\rangle^{2}\right]_{\mathrm{av}},
$$

where the average is over the bond realisations. $q$ is independent of the value of $\alpha$, (which runs from $\alpha=1$ to $M$ ). Under the MK iteration scheme the couplings flow to the high-temperature fixed point where the block spins are decoupled and only single site terms remain. As a consequence it is easy to evaluate $q$.

The Edwards-Anderson order parameter $q$ is plotted as a function of the reduced temperature in Fig. 3. The figure shows that $q \rightarrow 1$ as $T \rightarrow 0$ which is to be expected. At low temperatures where the correlation length is large both the $M=2$ and $M=3$ models behave almost identically, which is another example of the "universality" 


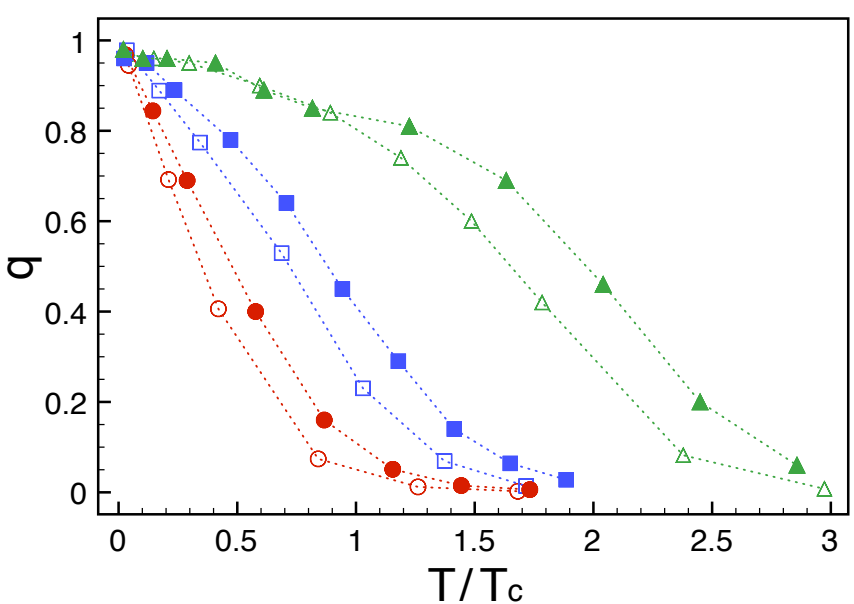

FIG. 3. (Color online) The Edwards-Anderson order parameter $q$ as a function of the reduced temperature. The symbols have the same association with $M$ and $d$ as in Fig. 1]

emerging in the problem.

What is striking is that $q$ is non-zero at any temperature, although it does become very small when $T \gg T_{c}$. $q$ is a measure of the extent to which the spin at site $i$ remembers its initial orientation: i.e.

$$
q=\frac{1}{N} \sum_{i}\left\langle\sigma_{i}^{(\alpha)}(0) \sigma_{i}^{(\alpha)}(t)\right\rangle, \quad t \rightarrow \infty .
$$

Thus in the $p$-spin models the spins never completely forget their initial orientations, no matter how high the temperature. This behavior is not a consequence of using the MK approximation. It is a feature which arises in any model described by the $\mathcal{H}_{\mathrm{GLW}}$ of Eq. (2) with a non-zero value of $w_{2}$, the term which breaks the timereversal symmetry. At mean-field level $q$ does vanish at temperatures above $T_{d}$.

$p$-spin models are meant to be useful models for understanding the properties of supercooled liquids so this feature of a non-vanishing $q$ is hard to reconcile with the properties of supercooled liquids. These forget their initial conditions after a time of the order of the alpha relaxation time, so for them $q$ is zero on long time scales. Maybe $p$-spin models are useful for describing the properties of supercooled liquids but only on timescales less than the alpha relaxation time. Given the huge effort which has gone into investigating their properties, this is certainly to be hoped.

Finally we note that if we had used the MK approximation with the further approximations which were made in Ref. [31, a Kauzmann transition would have been found for the model with $M=3$. It is only by carrying out the MK calculation exactly that one recovers the correct behavior [11].

JY was supported by WCU program through the KOSEF funded by the MEST (Grant No. R31-2008-00010057-0).
[1] T. R. Kirkpatrick and P. G. Wolynes, Phys. Rev. A 35, 3072 (1987); T. R. Kirkpatrick and D. Thirumalai, Phys. Rev. B 36, 5388 (1987); T. R. Kirkpatrick and P. G. Wolynes, Phys. Rev. B 36, 8552 (1987).

[2] V. Lubchenko and P. G. Wolynes, Annu. Rev. Phys. Chem. 58, 235 (2007).

[3] G. Biroli and J.-P. Bouchaud, arXiv:0912.2542

[4] W. Götze and L. Sjögren Rep. Prog. Phys 55241 (1992); W. Götze in Liquid, Freezing and the Glass Transition, edited by J. P. Hansen, D. Levesque and J. ZinnJustin, (Elsevier, New York, 1991); J. P. Bouchaud, L. F. Cugliandolo, J. Kurchan and M. Mezard in Spin Glasses and Random Fields, edited by A. P. Young, (World Scientific, Singapore, 1997).

[5] W. Kauzmann, Chem. Rev. 43, 219 (1948).

[6] M. Mézard and G. Parisi, Phys. Rev. Lett. 82, 747 (1999).

[7] G. Parisi and F. Zamponi, Rev. Mod. Phys. 82, 789 (2010).

[8] S. Franz, H. Jacquin, G. Parisi, P. Urbani, and F. Zamponi, cond-mat arXiv: 1206:2482.

[9] W. Kob, S. Roldán-Vargas, and L. Berthier, Nature Phys. 8, 164 (2012).

[10] M. A. Moore, Phys. Rev. Lett. 96, 137202 (2006).
[11] J. Yeo and M. A. Moore, Phys. Rev. B 85, 100405(R) (2012).

[12] B. Drossel, H. Bokil and M. A. Moore, Phys. Rev. E 62, 7690 (2000).

[13] G. Parisi, M. Picco and F. Ritort, Phys. Rev. E 60, 58 (1999).

[14] D. Larson, H. G. Katzgraber, M. A. Moore and A. P. Young, Phys. Rev. B 81, 064415 (2010).

[15] M. A. Moore and B. Drossel, Phys. Rev. Lett. 89, 217202 (2002).

[16] D. J. Gross, I. Kanter, and H. Sompolinsky, Phys. Rev. Lett. 55, 304 (1985).

[17] F. Caltagirone, U. Ferrari, L. Leuzzi, G. Parisi and T. Rizzo, Phys. Rev. B 83, 104202 (2011).

[18] E. Gardner, Nuclear Physics B 257 [FS14], 747 (1985).

[19] J. R. L. de Almeida and D. J. Thouless, J. Phys. A 11, 983 (1978).

[20] M. A. Moore and A. J. Bray, Phys. Rev. B 83, 224408 (2011).

[21] M. A. Moore, Phys. Rev. E 86, 031114 (2012).

[22] A. Cavagna, T. S. Grigera and P. Verrocchio, Phys. Rev. Lett. 98,187801 (2007).

[23] G. Biroli, J.-P. Bouchaud, A. Cavagna, T. S. Grigera and P. Verrocchio, Nature Phys. 4, 771 (2008). 
[24] D. S. Fisher and D. A. Huse, Phys. Rev. Lett. 56, 1601 (1986); Phys. Rev. B 38, 386 (1988); ibid. 38, 373 (1988).

[25] A. J. Bray and M. A. Moore, Lecture Notes in Physics 275, 121 (1986).

[26] W. L. McMillan, Phys. Rev. B 29, 4026 (1984).

[27] S. Boettcher, Phys. Rev. Lett. 95197205 (2005).

[28] M. A. Moore and J. Yeo, Phys. Rev. Lett. 96, 095701
(2006).

[29] M. Tarzia and M. A. Moore, Phys. Rev. E 75, 031502 (2007).

[30] C. Cammarota, G. Biroli, M. Tarzia, and G. Tarjus, cond-mat arXiv:1210.2941

[31] C. Cammarota, G. Biroli, M. Tarzia, and G. Tarjus, Phys. Rev. Lett. 106, 115705 (2011). 